\title{
Research on Distributed Wireless Network Capacity and Its Seepage Transmission Method
}

\author{
Bao Haining ${ }^{1, ~ *, ~ L u ~ J i h u a ~}{ }^{2}$ \\ ${ }^{1}$ Information Science Academy, China Electronics Technology Group Corporation, Beijing, China \\ ${ }^{2}$ School of Information and Electronics, Beijing Institute of Technology, Beijing, China
}

\section{Email address:}

baohaining@163.com (Bao Haining), bit_mail@163.com (Lu Jihua)

${ }^{*}$ Corresponding author

\section{To cite this article:}

Bao Haining, Lu Jihua. Research on Distributed Wireless Network Capacity and Its Seepage Transmission Method. Science Discovery. Vol. 5, No. 2, 2017, pp. 129-134. doi: 10.11648/j.sd.20170502.17

Received: March 31,2017; Accepted: April 18, 2017; Published: April 20, 2017

\begin{abstract}
Based on the background of green communication, the modeling and system capacity of distributed large-scale wireless networks have been deeply studied. We studied the robust rule of routing, capacity limit analysis, modeling of large-scale wireless network. and the system capacity. In detail, we focus on the modeling and connecting characteristics of large scale wireless network under the environment of dynamic link changes. Further, we analyze the existing capacity under the condition of multiuser interference and attenuation factors based on percolation theory. Finally, we propose the percolation routing and transmission method network based on the capacity limit of end-to-end wireless network system.
\end{abstract}

Keywords: Green Communications, Distributed Wireless Network, Capacity Analysis, Percolation Routing, Transmission Method

\section{分布式无线网络容量及其渗流传输方法的研究}

\section{鲍海宁 ${ }^{1 *}$, 卢继华 ${ }^{2}$}

${ }^{1}$ 信息科学研究院, 中国电子科技集团公司, 北京, 中国

'信息与电子学院, 北京理工大学, 北京, 中国

\section{邮箱}

baohaining@163.com（鲍海宁）, bit_mai1@163.com（卢继华）

摘要：基于绿色通信背景中的无线网络技术，本文深入研究了分布式大规模无线网络的路由存在准则、容量限分析、 网络建模及系统容量。具体针对链路动态变化的大规模无线网络的建模及连接特性, 考虑了多用户干扰、衰减因素存 在条件下的容量限分析, 再依据渗流原理建立基于网络容量限的端-端节点间的鲁棒多径路由, 最后探索了高效渗流传 输方法。

关键词: 绿色通信, 分布式无线网络, 容量分析, 渗流路由, 传输方法 


\section{1. 引言}

无线网络通信系统经历了巨大变革, 伴随着此变革, 在第 4 代的LTE出现了多址技术。据专家预测, 到 2020 年的 $5 \mathrm{G}$, 移动数据流量将有千倍的增长, 数据流量的爆炸性增 长将推动网络形式及模式进入新的矩形机器类通信时代 (massive machine-type-communications, mMTC) [1]。未 来的设备连接主要是通过无线网络, 据估计, 到2020年, 连接到互联网的设备将达到 500 亿。将近 $82 \%$ 的设备是通过 无线网络驱动的 [2]。

拟2020-2030年商用的下一代5G无线网络 NGN（Next Generation Network) 是以软交换为核心的分布式网络。 将会提供更高的容量且能量消耗比 $4 \mathrm{G}$ 系统更低 [3-4]。华 为公司在 “ $5 \mathrm{G}$ : A Technology Vision” 的技术报告中指 出了 $5 \mathrm{G}$ 无线网络系统有三大主要设计目标：1）实现极高 容量与超大规模互联；2）支持多样设备、应用与极多样 化的用户需求；3）异构网络下非连续频谱的灵活、高效 使用。

针对无线网络: 主要通过分布式系统配合灵活的站点 场景模型, 对无线网络建模、系统容量分析, 再对基站进 行改造以达到降低能量损耗的目的 [5]。绿色通信（Green Communications）这一通信理念, 具体指成本以及能量损 耗低的高效分布式无线通信系统。在具体系统设计方面, 主要体现在点到点无线通信系统以及无线网络系统 [5]。 为达这一目标, 需要有分布式接入网络, 其超高速、高可 靠的移动设备间传输能力、超密度异构网络下的绿色通信 目标, 十分令人期待 $[4-5][7-8]$ 。分布式容量计算一直是 多用户无线通信系统的研究热点, 因其容量系统设计能够 高效利用能量及传输资源, 实现绿色通信。

随着用户信息需求量、传输带宽及节点数日益增加, 分布式系统容量计算和评估也更为复杂 $[9-10]$ 。然而, 随 着移动通信领域的快速发展, 人们更希望了解分布式系统: 大规模无线网络的容量和逼近容限的通信方法 [11-12]。正 如Andrea Goldsmith所述, 人们对基本的网络容限研究十 分有限, 对如何达到和逼近网络容限知之更少 [13]。同时, 对于能够应用这一网络的通信方法, 人们还需研究其性能 以便更好的优化设计 [14]。

本文依据关于分布式XIXO-MU无线通信系统信道容量 的研究基础 [15-16], 结合渗流原理研究大规模无线网络容 量及系统设计 [17-18]。具体研究链路动态变化的大规模无 线网络建模及连接特性, 并基于多用户干扰及衰减因素分 析了分布式无线网络的容量性能。进一步研究大规模无线 网络容量、探索渗流路由与高效传输, 为甚高带宽、超多 用户数的未来无线通信网络的组网及传输提供设计依据。

\section{2. 分布式无线网络建模及连接特性分析}

\section{1. 分布式无线网络建模}

分布式无线网络构建与网络容量与覆盖、连通特性研 究密不可分, 是网络优化的重要课题和研究热点和难点 [20]。分布式无线网络构建在尺度和配对条件下, 密集型
网络 $G\left(\chi_{\rho}, g_{r_{\rho}}, A\right)$ 与扩展型网络 $G\left(\chi_{1}, g_{\sqrt{(\log \rho+b) / C}}, A_{\sqrt{\rho}}\right)$ 基于 模型 $G\left(\chi_{(\log \rho+b) / C}, g, A_{1 / r_{\rho}}\right)$ 是等效的, 且均可从无限网络模 型 $G\left(\chi_{(\log \rho+b) / C}, g, \Re^{2}\right)$ 中得到 $[14]$ 。因而, 渗流原理很适合 于研究复杂的分布式大规模网络。

分布式无线网络拓扑动态变化, 是指具有 “小世界” 特征分布式无线网络拓扑动态变化。在数学、物理学和社 会学中, 小世界网络是一种数学之图的类型, 在这种图中 大部分的结点不与彼此邻接, 但大部分结点可以从任一其 他点经少数几步就可到达。实际的社会、生态、等网络都 是小世界网络, 在这样的系统里, 信息传递速度快, 并且 少量改变几个连接, 就可以剧烈地改变网络的性能, 如对 已存在的网络进行调整, 如蜂窝电话网, 改动很少几条线 路, 就可以显著提高性能。对于规则网络, 任意两个点 (个 体)之间的特征路径长度长 (通过多少个体联系在一起), 但聚合系数高（你是朋友的朋友的朋友的几率高）。对于 随机网络, 任意两个点之间的特征路径长度短, 但聚合系 数低。而小世界网络, 点之间特征路径长度小, 接近随机 网络, 而聚合系数依旧相当高, 接近规则网络。发现规则 网络具有很高的聚合系数, 大世界 (large world, 意思 是特征路径长度很大), 其特征路径长度随着 $\mathrm{n}$ (网络中节 点的数量) 线性增长, 而随机网络聚合系数很小, 小世界 （small world, 意思是特征路径长度小）, 其特征路径 长度随着 $\log (\mathrm{n})$ 增长中说明, 在从规则网络向随机网络转 换的过程中, 实际上特征路径长度和聚合系数都会下降, 到变成随机网络的时候, 减少到最少。但这并不是说大的 聚合系数一定伴随着大的路径长度, 而小的路径长度伴随 着小的聚合系数, 小世界网络就具有大的聚合系数, 而特 征路径长度很小。

具有 “小世界” 特征, 其中众多移动节点间需自主连 接, 并需保证移动网络设备或节点间能实现高效而可靠的 通信。故需对系统中节点位置、功率分配及通信路径等做 假设, 以便于网络性能计算与传输方法设计。基于Poisson 分布建立随机图网络拓扑如下图1所示, 为便于观察选择 $10^{2}$ 个节点, 并依据选取规则 $\lfloor\operatorname{sqrt}(\operatorname{maxx} \times \operatorname{maxy} / 20)\rfloor$ 得 出, 其中, sqrt为开方运算, maxx和maxy分别是网络拓扑 面的长和宽, 实际处理的网络节点数应该规模为 $10^{4}$ 量级 [21]。

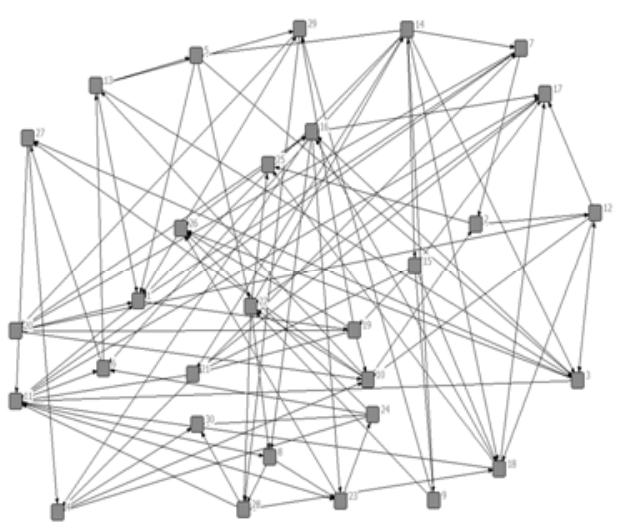

图1 基于随机几何模型建立分布式无线网络拓扑示意图。 
从图1可以看出, 网络节点是随机而归一化分布在长 和宽为 $\max x$ 和maxy的几何区域中, 且网络节点的拓扑分布 遵循泊松分布, 网络节点数的选取依据公式 $\lfloor\operatorname{sqrt}(\max x \times \operatorname{maxy} / 20)\rfloor 。$

\section{2. 分布式无线网络连接特性分析}

变换随机几何模型中半径参数得出网络中所有节点 的平均路径长度, 如图2所示。

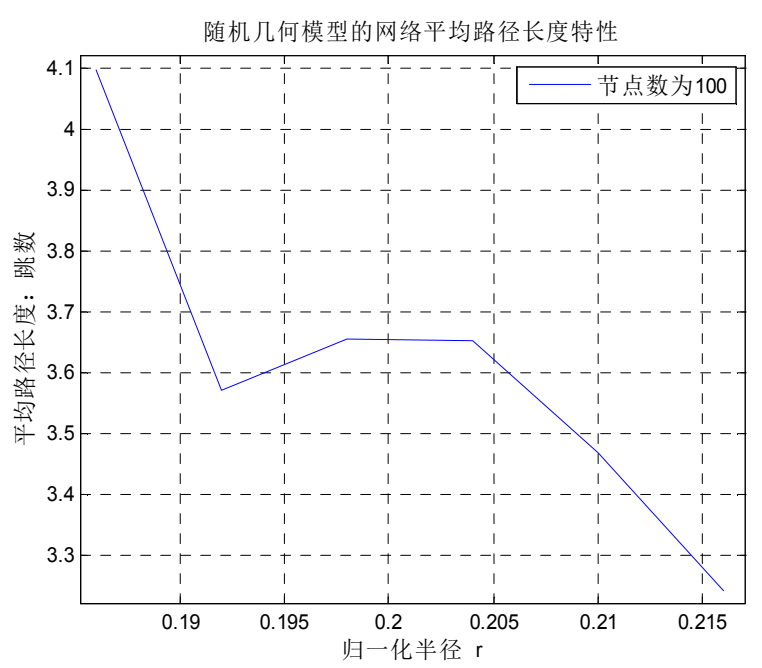

图2 随机几何模型网络的平均路径长度。

图2中, 横轴为归一化半径, 纵轴为平均路径长度, 即跳数。从图 2 可以看出, 归一化半径主要集中于 0.205 范围内, 且平局路径 长度集中于 3.6 跳以内。

由于网络曲率是衡量网络连通特性的重要指标, 是指 所有相邻节点之间连的数目占可能的最大连边数目的比 例, 系数越大说明能用小世界模型越接近 [22-23]。设网络 规模为 $10^{\wedge} 4$, 各自带有许多小而不相连的子图, 网络拓扑 设置接近实际网络规模且与实际值一致。对常用的九类随 机网络模型进行仿真, 得出各曲率值如表1所示:

表1 九类随机网络模型的曲率值.

\begin{tabular}{ll}
\hline 模型名称 & 曲率值 (C) \\
\hline Erdrey & $8.1009 \mathrm{e}-004$ \\
Gilbert & 0.0012 \\
Small world & 0.4616 \\
Geometric & 0.5825 \\
Pref & 0.0065 \\
Renga & 0.3925 \\
Kleinberg & 0.2001 \\
Lockandkey & 0.0021 \\
Sticky & 0.0134 \\
\hline
\end{tabular}

由表1中各网络模型曲率结果可知, Geometric网络模 型连通度最高, 曲率值高达0.5825, 而Smal1 world以及 Renga网络模型紧随其后, 曲率值分别为 0.4616 及 0.3925 。 因此，具有 “密集型” 特点的 “小世界特性” 的网络模型 连接度很高, 很适合构建大规模无线网络。

\section{3. 分布式无线网络容量分析}

2000年, Gupta与Kumar对 $\mathrm{n}$ 节点静态随机的ad hoc网 络容量进行研究, 给出节点吞吐容量随呈 $\mathrm{O}(1 / \sqrt{n})$ 减小的 结论 $[18]$ 。考虑衰减函数、容量尺度、干扰、延时及带宽 等各种因素的大规模网络容量研究取得了许多新进展 [11- 19]。但基于渗流原理针对分布式无线网络中节点协 作的网络容量研究还未见研究。

\section{1. 网络节点间最大传输速率分析}

在具有 $\mathrm{n}$ 个节点的分布式无线网络中, 假设这 $\mathrm{n}$ 个节点 均服从Poission分布在方形单位网络区域中, 带宽B很大, 则信号干扰功率比 (SINR) 可用式 (1) 表示:

$$
\operatorname{SINR}=\frac{W_{i j}\left|X_{i}-X_{j}\right|^{-\alpha}}{B N_{0}+\gamma \sum_{k \in I} W_{k}\left|X_{k}-X_{j}\right|^{-\alpha}}
$$

其中, $\gamma$ 表示正交因子, 表征CDMA网络中干扰的影响, 其变化范围是从 0 到 $1 ; N_{0}$ 为噪声功率, $\left|X_{i}-X_{j}\right|^{-\alpha}$ 表示节 点 $\mathrm{i}$ 到 $\mathrm{j}$ 的路径损耗, $W_{i j}$ 为节点 $\mathrm{i}$ 到 $\mathrm{j}$ 的传输功率; 路径损 耗可以表示为:

$W_{k}=\sum_{j} W_{k j} \leq W_{0}$, 其中, $W_{0}$ 为每节点发送的常功率。

根据香农公式对 (1) 关于 $B \rightarrow \infty$ 取极限, 得出节点 $i$ 和 $j$ 间的最大传输速率为:

$$
S h_{i j}=\lim _{B \rightarrow \infty} \mathrm{B} \log \left(1+\frac{W_{i j}\left|X_{i}-\mathrm{X}_{j}\right|^{-\alpha}}{N_{0} B+\gamma \sum_{k \in I} W_{\mathrm{k} j}\left|X_{k}-\mathrm{X}_{j}\right|^{-\alpha}}\right)
$$

依据极限定理, 上式的最大传输速率为可以进一步表 示成如下公式 (2):

$$
S h_{i j}=\frac{W_{i j}\left|X_{i}-\mathrm{X}_{j}\right|^{-\alpha}}{N_{0}}
$$

\section{2. 基于渗流原理推导CDMA网络的多址容量}

基于网格的渗流模型中边长度为 $\mathrm{c}_{0} \sqrt{n}$, 每网格至少 包含 1 个节点的概率可以表达为式 (3):

$$
p=1-\mathrm{e}^{-\mathrm{c}_{0}^{2}}
$$

假设有节点以Sh (n) nat/s传输到目的端, 随机选取 一对节点, 归一化吞吐量取决于路由及调度机制。已有文 献证明 [21]：优化路由、最大化吞吐量的前提条件是每一 对源宿节点对具有最小的功率路由。

因此: 最小化路由的功率消耗等价于所有节点平均功 率的最小值。对于给定的第 $\mathrm{i}$ 对节点, 其路由为: $R_{i}=\left[\mathrm{X}_{i_{1}}, \mathrm{X}_{i_{2}}, \ldots, \mathrm{X}_{i_{K}}\right], S h_{i}$ 表示路径 $R_{i}$ 的吞吐量, 可推导出 此路径的最小功率可以表达为如下公式 (4): 


$$
\mathrm{W}\left(\mathrm{R}_{i}\right)=\mathrm{Sh}_{i}(\mathrm{n}) \cdot \mathrm{N}_{0} \sum_{k=1}^{K-1}\left|X_{i_{k}}-\mathrm{X}_{i_{k+1}}\right|^{\alpha}
$$

其中, $\mathrm{Sh}_{i}(\mathrm{n})$ 来源于公式 (2), 表示整个 $R_{i}$ 路由可获 得的吞吐量, 即最大传输速率。假设源目的节点对间至少 有长为 $m$ 的路径, 则 $m$ 值为:

$$
\mathrm{m}=\frac{\mathrm{d}_{i}}{\sqrt{2} c_{0} / \sqrt{n}}=\frac{\mathrm{d}_{i} \sqrt{n}}{\sqrt{2} c_{0}}
$$

其中, $\sum_{k=1}^{K-1}\left|X_{i_{k}}-\mathrm{X}_{i_{k+1}}\right|$ 为功率速率 $Q_{i}$, 即:

$$
Q_{i}=\sum_{k=1}^{K-1}\left|X_{i_{k}}-X_{i_{k+1}}\right|^{\alpha}
$$

前述已知: 对于源节点与宿节点对间的每个栅格而言, 第一跳最多 8 种选择, 则其后的跳数最多有 7 种选择, 且每 个路径的开概率为 $\mathrm{p}^{\mathrm{m}}$ 。因此, $\mathrm{m}$ 条开路径的数学期望可表 达为如下Markov不等式:

$$
\mathrm{P}(\mathrm{N}(\mathrm{m}) \geq 1) \leq E[\mathrm{~N}(\mathrm{~m})]=\frac{8}{7}(7 \mathrm{p})^{\mathrm{m}}=\frac{8}{7}(7 \mathrm{p})^{\frac{\mathrm{d}_{\mathrm{i}} \sqrt{n}}{\sqrt{2 c_{0}}}}
$$

其中, $\mathrm{N}(\mathrm{m})$ 为长 $m$ 的开路径数, 即对于任意从源到目 的节点的一对节点之间, 可建立若干跳组成的路径。

\section{3. m跳网络路径功率速率概率及网络容量上限分析}

对于 $T^{*}$ 上每一个闭的网格, 至少存在一个长度 $\geq \mathrm{c}_{0}^{\prime} / \sqrt{n}$ 的功率路由; 若一个链路涉及了 $T^{*}$ 上的 $\mathrm{j}$ 个网格, 其功率速率应该更大, 因此在 $\mathrm{c}_{3} \sqrt{n}$ 链路内存在概率 $\geq \mathrm{p}_{1}$, 长度 $\geq \mathrm{c}_{0}^{\prime} / \sqrt{n}$ 的路径。

根据公式 (6) 可得出功率速率为 $c_{3} \sqrt{n} \cdot\left(\frac{c_{0}^{\prime}}{\sqrt{n}}\right)^{\alpha}=c_{3} c_{0}^{\prime \alpha} n^{(1-\alpha) / 2}$, 令 $c_{1}=c_{3} c_{0}^{\prime \alpha}$, 得出:

$$
\mathrm{P}\left(\mathrm{Q}_{i}>c_{1} n^{(1-\alpha) / 2}\right) \geq p_{1}=1-\frac{8}{7} \exp \left(-c_{2} \sqrt{n}\right)
$$

合并 (4) 和 (6), 可得:

$$
\mathrm{W}\left(\mathrm{R}_{i}\right)=\mathrm{Sh}_{i}(\mathrm{n}) \cdot \mathrm{N}_{0} Q_{i}
$$

设 $\mathrm{R}^{\varepsilon}$ 代表源和目的节点对间距离至少为 $\varepsilon$ 的路由 $R_{i}=\left[\mathrm{X}_{i_{1}}, \mathrm{X}_{i_{2}}, \ldots, \mathrm{X}_{i_{K}}\right]$ 集合; 由文献 [21] 中定理和推导可知 $n / 8 \leq\left|\mathrm{R}^{\varepsilon}\right| \leq 2 \mathrm{n}$ 。将 $\mathrm{R}^{\varepsilon}$ 中所有路由取和得:

$$
\sum_{i \in R^{\varepsilon}} \mathrm{W}\left(\mathrm{R}_{i}\right)=\sum_{i \in R^{\varepsilon}} \mathrm{Sh}_{i}(\mathrm{n}) \cdot \mathrm{N}_{0} Q_{i} \geq \mathrm{Sh}(\mathrm{n}) \sum_{i \in R^{\varepsilon}} \mathrm{N}_{0} Q_{i}
$$

公式(1) 又可表达为:

$$
\mathrm{P}\left(\mathrm{Q}_{i} \leq c_{1} n^{(1-\alpha) / 2}\right) \leq \frac{8}{7} \exp \left(-c_{2} \sqrt{n}\right)
$$

不失一般性, 可进一步得到:

$$
\mathrm{P}\left(\exists \mathrm{i} \in \mathrm{R}^{\varepsilon}, \text { s.t. } \mathrm{Q}_{i} \leq c_{1} n^{(1-\alpha) / 2}\right) \leq 2 \mathrm{n} \cdot \frac{8}{7} \exp \left(-c_{2} \sqrt{n}\right)
$$

每条路由功率 $\geq c_{1} n^{(1-\alpha) / 2}$, 所有 $2 \mathrm{n}$ 条路由功率最大为: $2 \mathrm{nW}_{0}$, 将其代入公式 (10) 得:

$$
\operatorname{Sh}(\mathrm{n}) \leq \frac{2 W_{0}}{N_{0} c_{1} n^{(1-\alpha) / 2} / 8}=c_{4} n^{(\alpha-1) / 2}
$$

至此完成了考虑了正交性、干扰及功率衰减等因素的 无线网络容量上限的推导, 同时也给出了分布式无线网络 的功率速率、传输速率及并行路由数量的设计思路。

\section{4. 鲁棒多路径路由建立及渗流传输方法}

\section{1. 多跳并行路由存在的条件}

上节所述的 “节点对间高概率路由建立” 是分布式无 线网络协议设计与高速数据传输的理论基础。基于前述无 线网络容量推导可得多跳并行路由存在的条件: 渗流网络 中至少可构建 $n / 8$ 条并行路由, 且每条路由功率至少为 $c_{1} n^{(1-\alpha) / 2}$ 。

至于关于如何实现多条并行路由, 有学者提出了源节 点到目的节点间能容忍链路失败或消失的鲁棒多径 (也称 为 “编织路由”) 传输机制 [24]。以最小代价实现在节点 失效和链路变化情况下提供鲁棒传输, 构建 $\mathrm{k}$-跳多径路由 (所有节点最短跳数为 $\mathrm{k}$, 且使得 $\mathrm{k}$ 尽量小) 情况下建立 “渗 流”来对抗链路和节点 $\mathrm{b}$ 失败, 从而实现高速率传输 [25-26]。

\section{2. 基于渗流原理构建分布式无线网络}

基于渗流原理构建网格数为 $m \times \log m$ 边长为 $1 / m=c_{5} / \sqrt{2 n}$ 的分布式无线网络 [19]。其中, $\mathrm{n}$ 为网络密 度 (网络节点数), 网络中节点可以可靠通信的前提是两节 点间距小于 $r$, 假设半径满足: $r \geq 2 \sqrt{2} c_{0}$; 为了更好捕获 节点失败, 假设每个节点独立, 且拥有不同存在概率以描 述节点位置不确定和捕获节点失败。设源节点为 $\mathrm{s}$ 和目的 及节点为 $\mathrm{d}$, 依据上述 3.3 中关于无线网络容量的上界推导, 可实现节点间距为 $r$ 的半径范围内的多跳可靠通信。

\section{3. $\mathrm{m}$ 跳路径建立事件A及路由形成概率推导}

我们定义 $T^{*}$ 包含 $\geq \mathrm{c}_{3} \sqrt{n}$ 条关到开的网格, 可获得一 条 $\mathrm{m}$ 跳路径, 设 $\mathrm{A}$ 为从源开始的 $\mathrm{m}$ 跳路径, $\mathrm{F}_{A}$ 为 $\mathrm{A}$ 事件发生需 打开最少数量的闭网格, 且: $\mathrm{F}_{A} \leq \mathrm{c}_{3} \sqrt{n}$ 。

$$
\mathrm{P}_{p^{\prime}}\left(\mathrm{F}_{A} \leq \mathrm{c}_{3} \sqrt{n}\right) \leq \frac{8}{7}(7 \mathrm{p})^{\mathrm{m}}\left(\frac{p-p^{\prime}}{1-p^{\prime}}\right)^{-c_{0} / \sqrt{n}}
$$


其中, $p^{\prime}=\frac{k p-1}{k-1}<p$, 且 $k>1 / p$; 将式 (3) 代入 (8), 推导得:

$$
\begin{aligned}
& \mathrm{P}_{p^{\prime}}\left(\mathrm{F}_{A} \leq \mathrm{c}_{3} \sqrt{n}\right) \leq \frac{8}{7}(7 \mathrm{p})^{\frac{\mathrm{d}_{1} \sqrt{n}}{\sqrt{2} c_{0}}} \cdot k^{c_{3} \sqrt{n}} \\
& =\frac{8}{7} \cdot \exp \left(\sqrt{n}\left(\frac{\mathrm{d}_{i} \log (7 p)}{\sqrt{2} c_{0}}+c_{3} \log k\right)\right) \\
& =\frac{8}{7} \cdot \exp \left(-c_{2} \sqrt{n}\right)
\end{aligned}
$$

其中, $c_{2}=-\frac{\mathrm{d}_{i} \log (7 p)}{\sqrt{2} c_{0}}-c_{3} \log k$ 。

（15）式又表明 $T^{*}$ 中包含至少 $\mathrm{c}_{3} \sqrt{n}$ 条路径的概率可以 表达为:

$$
p_{1}=1-\frac{8}{7} \cdot \exp \left(-c_{2} \sqrt{n}\right)
$$

\section{4. 探针机制建立多跳路由}

步骤一、源节点 $s$ 向其近邻节点发送探针包; 探针包 中包含源节点地址信息、目的节点地址信息和转发次数计 数器, 并预留 $\mathrm{Y}$ 个转发节点地址的位置, 其中转发次数计 数器置零;

步骤二、近邻节点接收到的探针包后, 如果本节点为 目的节点, 转步骤三; 否则本节点作为中间节点检查转发 次数计数器的值, 若该值小于 $Y$ 时存在两种情况:

步骤2.1如果本节点存在没有闭环的近邻节点, 探针 包转发次数计数器加 1 , 将本节点地址写入探针包, 将更 新后的探针包转发到本节点的近邻节点中可达目的节点 度量最大的近邻节点, 重复步骤二, 直到所转发的近邻节 点为目的节点时, 转步骤三;

步骤2.2如果不存在没有闭环的近邻节点, 则丢弃此 探针包, 结束本次选路过程;

步骤三、目的节点根据从源节点到目的节点各探针包 中所包含的路径信息, 使用路径保留准则计算并保留其中 至多X条并发路径信息; 所谓路径保留准则是指探针包所 探测的源节点 到目的节点的通路大于X时, 只保留路径最 短或者吞吐能力最大的X条路径信息;

步骤四、目的节点 $\mathrm{d}$ 将选中的路径信息组成数据包, 分别通过各路径信息中的路由反馈给发送端; 反馈信息通 过各中间节点时, 各中间节点增加本中间节点可达该路由 上其他节点的单位度量值更并新自己的其他路由信息。

\section{5. 分布式无线网络的高效渗流传输方法}

在实际网络中, 基于概率转发的路由, 其部分数据包 可能是由于路由中断、链路差错和丢包等原因, 无法传送 到目的节点, 这些都会造成源数据无法恢复的情况发生。

本文基于渗流理论研究二维泊松格状网络模型中节 点间高概率路由形成与传输, 提出了针对分布式无线网络 的渗流传输方法: 包含建立路由阶段和数据传输阶段。其 中, 在建立路由阶段中探针包在网络中传输基于探针机制
寻找多跳并行路由, 渗流理论用于确立多条并行路由的存 在条件。

在数据传输阶段, 发送节点将数据进行喷泉编码后拆 分, 再基于实时信道信息以自适应速率在网络中传输。喷 泉编码保证了数据传输的可靠性, 即使网络中部分分组丢 失, 只要目的节点接收到足够的数据包, 也可以采用解码 的方法恢复源数据, 无需反馈重传。

\section{5. 结论}

本文基于渗流原理, 着重研究了分布式无线网络高效 和高可靠传输问题, 推导了无线网络容量限, 并架设起渗 流原理、随机图论概率论及数据传输方法设计与性能评估 之间的桥梁。对人们以前研究较少的分布式网络性能、渗 流原理引发的传输方法进行分析, 基于理论推导与仿真分 析分布式无线网络的构建思路和逼近网络容限的新型传 输方法, 具体工作如下:

首先, 基于密集型与扩展型网络特点, 研究分布式无 线网络模型的建立方法, 搭建“小世界”特性的无线网络, 并研究网络簇系数与平均路径长度特性;

其次, 分析衰减、干扰等约束条件下的分布式无线网 络的连通性与信道容量, 并进一步推导大规模无线网络的 容量限。

最后, 在连通性、容量计算与推导基础上, 分析了多 跳路由的形成条件及路由形成概率, 并基于探针机制建立 分布式复杂网络的并行鲁棒路由, 然后给出了高效渗流传 输方法。

\section{参考文献}

[1] ABIresearch, [0nline] https://www. abiresearch. Com The Internet of Things Will Drive Wireless Connected Devices to 40.9 Billion in 2020.

[2] IMT-2020 PG, “IMT Vision towards 2020 and beyond, ” ITU-R WP5D ITU-R 2020 Vision Workshop, February 12th, 2014.

[3] Chih-Lin I, Shuangfeng H, Zhikun X, et al. Towards green and soft: $5 \mathrm{G}$ design considerations $[\mathrm{J}]$. Australian Journal of Telecommunications and the Digital Economy, 2014, 2(3): 48.

[4] C. I, C. Rowell, S. Han, et. al. "Toward Green and Soft: A 5G Perspective, ” IEEE Commun. Magazine, Vol. 52, no. 2, 66-72, Feb. 2014.

[5] 3GPP TS 36.213 version 13.0.0, “Evolved Universal Terrestrial Radio Access (E-UTRA); Physical layer procedures, ” 3GPP TSG RAN, 2016.

[6] Z. Yuan, G. Yu, W. Li, Y. Yuan, X. Wang and J. Xu, "Multi-user shared access for Internet of things, ” IEEE Vehicular Tech. Conf. (VTC Spring), pp. 1-5, 2016. 
[7] Rowell C, Han S, Xu Z, et al. Green RF technologies for $5 \mathrm{G}$ networks[C]//Wireless Symposium (IWS), 2014 IEEE International. IEEE, 2014: 1-4.

[8] B. Dai and W. Yu, “Energy efficiency of downlink transmission strategies for cloud radio access networks, ” IEEE Journal on Selected Areas in Communications, vol. 34, no. 4, pp. 1037-1050, 2016.

[9] W. Cheng, X. Zhang, and H. Zhang, “Statistical-QoS driven energy-efficiency optimization over green $5 G$ mobile wireless networks, ” IEEE Journal on Selected Areas in Communications, vol. 34, no. 12, pp. $3092-$ 3107, 2016.

[10] T. S. Rappaport, Wireless communications: principles and practice, Second edition, [M]. Prentice Hall, 2002.

[11] 陈晓明, 张朝阳, 王超. “多用户分布式与集中式天线系统 传输容量的分析与比较” [J]. 中国科学技术大学学 报, Vol. 39, no. 10, 2009, pp. 1097-1101。

[12] P. Gupta and P. R. Kumar. "The capacity of wireless networks " [J]. IEEE Transactions on Information Theory, Vol. 46(2), Mar. 2000, pp. 388-404.

[13] S. Lee; S. Moon; J. Kim; I. Lee, “Capacity Analysis of Distributed Antenna Systems in a Composite Fading Channel " [J]. IEEE Transactions on Wireless Communications, Vol. 11, no. 3, 2012, pp. 1076-1086.

[14] Andrea Goldsmith, "Capacity, cooperation, and cross-layer design in wireless networks " [C]. Conference on Information Sciences and Systems. Princeton, March 24, 2006.

[15] Guoqiang Mao and Brian D. 0. Anderson, “Towards a Better Understanding of Large-Scale Network Mode1s” [J]. IEEE/ACM Transactions on Networking, 2012, Vol. 20, No. 2 , pp. $408-421$.

[16] W. Choi, J. G. Andrews. "Downlink performance and capacity of distributed antenna systems in a multicell environment, " [J]. IEEE Transactions on Wireless Communications, Vol. 6, No. 1, Jan. 2007. pp. 69-73.
[17] Ai. Wang and J. Lu. “An Efficient Algorithm for Performance Analysis of Multi-cell and Multi-user Wireless Communication Systems ” [J]. KSII Transactions on Internet and Information Systems, Nov. 2011, pp. 2035-2051.

[18] S. R. Broadbent and J. M. Hammersley, "Percolation processes I. Crystals and mazes” [C]. Proceedings of Cambridge Philosoph. Soc., 1957, Vol. 53, pp.629641.

[19] 0. Dousse, F. G. Grimmett, Percolation, Second ed. [M]. New York: Springer-Verlag, 1999.

[20] Chi-Kin Chau, Richard J. Gibbens, Robert E. Hancock, et al. "Robust multipath routing in large wireless networks" INFOCOM IEEE Conference on Computer Communications Workshops, 2011, pp. 271-275.

[21] Dousse 0, Thiran P. “Connectivity vs Capacity in Dense Ad Hoc Networks ” IEEE Infocom 2004 Proceedings [C]. 2004, pp. 486-495.

[22] 01ivier Dousse, Fran cois Baccelli, and Patric Thiran. "Impact of Interferences on Connectivity in Ad Hoc Networks", IEEE/ACM Transactions on networking, Vol. 13, No. 2, April 2005, pp. 425-436.

[23] F. Ariaei, M. Lou, E. Jonckheere, B. Krishnamachari, and M. Zuniga, "Curvature of Indoor Sensor Network: Clustering Coefficient" EURASIP Journal on Wireless Communications and Networking, 2008, pp. 1-20.

[24] R. Negi and A. Rajeswaran. "Capacity of power constrained ad-hoc networks” [J]. In Proc. Of IEEE Infocom 2004, March 2004.

[25] D. Ganesan, R. Govindan, S. Shenker, and D. Estrin, " Highly-resilient, energy-efficient multipath routing in wireless sensor networks " Mobile Computing and Communications Review, vol. 4, no. 5, 2001.

[26] V. Manfredi, R. Hancock, and J. Kurose, “Robust routing in dynamic manets" Department of Computer Science, University of Massachusetts, Amherst, Tech. Rep., 2009. 